comprises an editorial overview of up to 16 reviews by recognized experts in the field, combining timely assessments of accumulating data with occasionally controversial but always interesting points of view.

The papers are generally of high quality, well written, beautifully illustrated and fully referenced (with, usefully, brief résumés of key features of a few of the more important citations). The journal is not, of course, a substitute for actually going and reading the primary data in the original papers. But it does give the overworked, overwhelmed microbiologist an invaluable, authoritative and convenient way of keeping up to date with a familiar field or of beginning to come to grips with an unfamiliar one. And by revisiting each aspect annually, the editors have ensured a regular 'state of the nation' appraisal that will help microbiologists make sense of the information explosion.

Peter H. Williams is in the Department of Microbiology and Immunology, University of Leicester, Leicester LE1 9HN, UK.

\section{Computers and the human brain}

\section{CyberPsychology \& Behavior}

Editor Mark D. Wiederhold

Mary Ann Liebert. 6/yr. USA \$143, elsewhere $\$ 182$ (institutional); USA $\$ 79$, elsewhere $\$ 119$ (personal)

\section{Amanda Parker}

Separating the interesting issues related to the impact of the Internet, multimedia and virtual reality (VR) from their respective media hype is never going to be easy. Like these products of the global information age, scientific research into their effects on behaviour and society is likely to be both prolific and of a very variable quality. The challenge for a journal devoted to this area, then, is likely to be one of selection.

The range of articles in CyberPsychology $\&$ Behavior $(C \& B)$ is wide. The journal is primarily aimed at healthcare providers who are interested in the use of advanced technology to reduce the cost of healthcare. Consequently, many articles discuss advances in virtual reality that allow its application in a mental health setting. For example, patients with acquired brain injuries, neurological disorders and developmental disabilities may benefit from VR training in a range of simulated environments. VR also has exciting possibilities in psychotherapy. Phobias may be treated with more precision in a virtual environment which can be switched off when necessary: recently published $C \& B$ articles show that fear of flying is one area in which progress is being made. The benefits of VR in treatment of post-traumatic stress

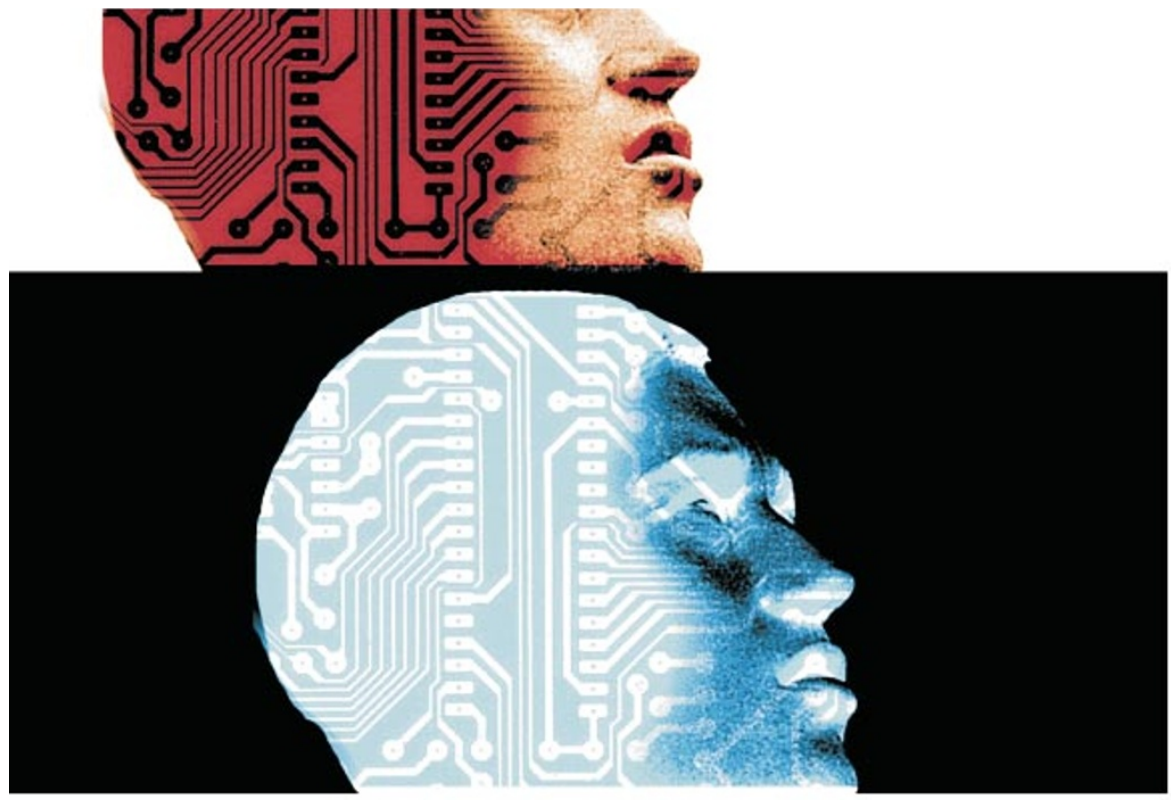

disorder have also been recently discussed.

On the other hand, evidence of the Internet's value in psychotherapy is less convincing, and articles presenting research into the Internet's impact on behaviour tend to rely on opportunity sampling and number crunching to reach their conclusions. These papers take a very different approach from the patient studies discussed above, which makes for an uneasy balance in the journal. CyberPsychology \& Behavior provides a forum for a range of topics that may turn out to be too wide for comfort.

Amanda Parker is at the School of Psychology, University of Nottingham, University Park, Nottingham NG7 2RD, UK.

\section{Testing the market in search of a niche}

\section{Genetic Testing}

Editor Fred Gilbert

Mary Ann Liebert. 4/yr. USA \$186,

elsewhere \$238 (institutional).

USA \$99, elsewhere $\$ 150$ (personal).

USA \$50, elsewhere $\$ 75$ (student)

\section{Peter Harper}

Although I had seen the initial publicity for this new journal, it was interesting to see how the first 18 months of production has matched up to the initial stated aims. The journal has a distinguished molecular geneticist as editor-in-chief and a broadranging, well-respected editorial board (though it is not clear whether they are functional rather than decorative).

The editorial in the first issue makes it clear that Genetic Testingis intended to have a wide readership including the professional genetic testing community, those who develop or perform such testing, and those "whose expertise and interests fall within the scope of utilization, interpretation, and delivery of genetic testing." The journal invites original papers on diagnostic genetic testing of all kinds, newborn and carrier screening, technical approaches to testing and ethical and social aspects.

The balance of papers is very much towards reviews, with several collections of papers resulting from symposia occupying most of the space. The first of these, spread over two issues, is a series of reviews on the frequency of genetic disorders and mutations in Jewish populations, concentrating on disorders of specific significance in Ashkenazi Jews. This is a good example of material that is often scattered too widely or not published at all, so it is good to see the collection appear here, though one might ask whether it would not find a wider readership in a small monograph.

The second collection occupying an entire issue relates to genetic testing and Alzheimer's disease. Again the broad conclusions had appeared earlier (in Nature Medicine 7, 757-759; 1998) but there is much detail in addition that deserves publication.

The third collective series is on the topic of genetic privacy. I found this less valuable, perhaps largely because many contributions, particularly those on insurance and genetic tests, were parochial and written in the specific context of the United States rather than internationally.

In terms of original articles, I do not think that any of the papers I saw rate highly, nor would I consider the technical reports particularly important. There are some disease maps of the human genome which seem rather redundant now that databases cover this information and are regularly updated.

It would be nice to think that scientists, genetic counsellors and ethicists as well as 
others might all be readers and gain from the interactions, but I rather doubt whether this will actually happen. I suspect that this may become a journal more related to clinical practice and its social and ethical implications than to high-profile original contributions. Though this may not have been the original intention behind the journal, it could turn out to be the right course; perhaps it indicates a niche that has not been filled by other journals so far.

My advice to the editors would be to encourage the journal to develop along whichever direction it looks like taking and not be too worried if it fails to attract highprofile original work. There should be plenty of opportunities for useful contributions over the next few years.

Peter Harper is at the Institute of Medical Genetics, University of Wales College of Medicine, Heath Park, CardiffCF4 4XN, UK.

\section{n......................... Evolving discipline finds a forum}

\begin{tabular}{l} 
Nutritional Neuroscience \\
Editor Chandan Prasad \\
Harwood Academic. 6/yr. 86 euros \\
\hline Public Health Nutrition \\
Editor Barrie Margetts \\
CABI. $4 / y r . £ 175, \$ 310$ (institutional); $£ 48$, \\
$\$ 80$ (special rate for members of The \\
Nutrition Society) \\
Gema Frühbeck
\end{tabular}

Given the cornucopia of nutrition literature, one could justifiably ask if we need yet another journal in this field. Nutrition in the twenty-first century will be based increasingly on creating an interdisciplinary field of epidemiology, structural biology, integrative physiology and molecular pathogenesis. This should ensure that we revolutionize the ways in which we investigate, diagnose and treat nutrition-related health problems.

The late twentieth century has seen a number of fundamental transformations in the study of the brain. Recently, the qualitative approach crucial to understanding nutrition has
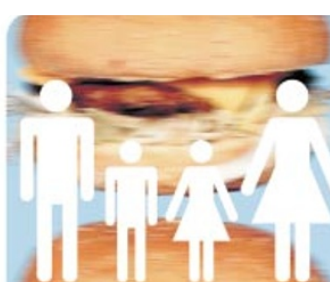

been embraced by neuroscientists to the extent that the subject may be on the verge of a golden age, in which fields once thought impenetrable can be explored in detail. The publishing venture of Nutritional Neuroscience is an acknowledgement of the growing community of researchers dedicated to studying these very exciting topics from a wide range of disciplinary perspectives. The enterprise will play an important part in bridging the gap between neuroscientists and nutritionists by focusing on eating disorders, at the same time as trying to unravel the complex underlying neuroanatomical and pathophysiological processes.

Given the Jeremiahs who prophesy that it is foolish to launch journals in such difficult financial times, the publishing venture of two new nutrition-oriented journals must be congratulated.

Nutritional Neuroscience is a handsome periodical covering an admirably broad range of topics including the effects of the various components of diet, dietary supplements and food additives on neurochemistry, neurobiology and behavioural biology. The neural and hormonal control of food intake, and dietary considerations in the management of neurologic and psychiatric disorders are also covered. It is the first journal to focus specifically on this area, and fills an important niche. The journal therefore merits reading by neuroscientists, nutritionists, neurologists and psychiatrists.

Public Health Nutrition was launched in March last year to cover studies involving nutritional epidemiology, nutrition-related health promotion, evaluation of the effectiveness of intervention studies aimed at improving health, the role of nutrition in high-risk and vulnerable groups as well as population-based research related to primary prevention of illness. This periodical will be valued by nutritionists and dietitians involved in nutritional epidemiology research, primary prevention and public health, together with epidemiologists and health-promotion specialists who are interested in the increasingly recognized role played by nutrition in disease prevention.

This attractive journal has an outstanding editorial board, with members representing 11 countries and committed to supporting, developing and maintaining an international perspective. So far, about half of the articles have come from Europe, the United States and Canada. Interestingly, 39 per cent of articles have been submitted by groups from different countries working in collaboration.

The period from submission to acceptance is generally two to three months, although the lag for actual publication is around five to six months. Public Health Nutrition fulfils the requisites of a high-quality journal and is therefore most welcome.

Web-surfing scientists may also enjoy visiting the electronic address of The Nutrition Society (http://www.nutsoc.org.uk/ phnj) to browse through the papers of all of the issues of Public Health Nutrition. Updated regularly, the site is extremely user-friendly. Gema Frühbeck is in the Department of Endocrinology, Clinica Universitaria de Navarra, University of Navarra, 31008 Pamplona, Spain.

\section{Birds unlimited}

BWP Update: The Journal of Birds of the Western Palearctic

Editor Malcolm Ogilvie

Oxford University Press. 3/yr. Print only. $£ 85$, $\$ 150$ (institutional); $£ 45, \$ 75$ (personal)

\section{Juha Merilä}

Ornithology is in a unique position among the biological sciences: practitioners are, with ever-increasing pace and in meticulous detail, being deluged with new literature about the lives and appearances of their study objects. Caught in this explosion of information, the relatively recent (1977-94), nine-volume, 7021-page reference work, Handbook of the Birds of Europe, the Middle East and North Africa (Oxford University Press, $\mathfrak{E} 795$ ), also known as Birds of the Western Palearctic (BWP), is already outdated in many places. A new journal, BWP Update, was therefore launched to keep this ambitious and authoritative work as comprehensive as possible.

Since its launch in April 1997, BWP Update has covered 30 species (out of the 797 in $B W P$ ), including short descriptions for 10 species new to the Western Palearctic. The style and format of the book are largely maintained, but a new section on conservation has been added. Distribution maps are now re-drawn and updated in colour.

With its lavish and well-edited appearance, BWP Update may appeal to both professional and amateur ornithologists and birdwatchers, although many may be put off by its high subscription price - especially since much of the 'new information', such as updates on population trends and distribution ranges, has already appeared in better formats in recent books. On reflection, an easily updateable and searchable online/ CD-ROM edition of BWP Update would be a more valuable innovation than this journal for those using $B W P$ on a regular basis.

Juha Merilä is in the Department of Population Biology, Evolutionary Biology Centre,

Uppsala University, Norbyvägen $18 d$,

SE-75236, Uppsala, Sweden.

\section{Depends how much time you've got}

\section{Mechanics of Time-Dependent} Materials

Editors Wolfgang Knauss and Igor Emri Kluwer. 4/yr. DFl525, \$262

\section{David P. Pope}

It seems only yesterday that we were all decrying the proliferation of journals (the comment by one of my colleagues some 